\title{
Procrastinación en Universitarios: Evidencia Preliminar del Efecto de Contextualización en Asignaturas Específicas
}

\author{
Sergio Dominguez-Lara \\ Universidad de San Martín de Porres, Lima, Perú
}

\section{RESUMEN}

La contextualización de tests psicológicos en cursos específicos es necesaria para obtener informaciones relevantes, y sustentar las intervenciones individuales o grupales, a diferencia de los instrumentos de evaluación general, que se concentran en la vida académica global. La procrastinación académica es relevante, y su evaluación es necesaria debido a sus consecuencias a mediano y corto plazo. Por lo tanto, el objetivo del presente informe metodológico fue comparar el resultado del análisis estructural, relación entre las dimensiones, la confiabilidad y las especificaciones erróneas de la Escala de Procrastinación Académica, evaluada en su forma general (muestra 1: 717 estudiantes, 74,3\% mujeres), y demarcada en asignaturas específicas (muestra 2: 142 estudiantes; 72,5\% mujeres). Los resultados indican que la forma específica tiene mayor respaldo psicométrico en el ajuste global, relación entre factores, y menor frecuencia de especificaciones erróneas. Sin embargo, los coeficientes de confiabilidad no difieren entre las versiones. Se discuten las implicancias de los resultados.

Palabras clave: procrastinación académica; validez; confiabilidad; especificaciones erróneas; asignaturas específicas.

\section{RESUMO - Procrastinação em Universitários: Evidências Preliminares do Efeito da Contextualização em Cursos} Específicos

A contextualização de testes psicológicos em cursos específicos é necessária para obter informações relevantes para sustentar intervenções individuais ou grupais, diferente dos instrumentos de avaliação geral, concentrados na vida acadêmica global. A procrastinação acadêmica é relevante, e a sua avaliação é necessária por suas consequências a curto e médio prazo. Então, o objetivo do presente reporte metodológico foi comparar o resultado da análise estrutural, relação entre as dimensões, a confiabilidade e as especificações erradas da Escala de Procrastinação Acadêmica, avaliada em sua forma geral (amostra 1: 717 estudantes, 74.3\% mulheres) e demarcada em assinaturas específicas (amostra 2: 142 estudantes, 72.5\% mulheres). Os resultados indicam que a forma específica tem maior respaldo psicométrico, tanto no ajuste global, relação entre fatores, e menor frequência de especificações erradas. No entanto, os coeficientes de confiabilidade não diferem entre as versões. As implicâncias dos resultados são discutidas.

Palavras-chave: procrastinação académica; validade; fiabilidade; especificações erradas; assinaturas específicas.

\section{ABSTRACT - Procrastination in College Students: Preliminary Evidence of Effect of the Frameworking in Specific Subjects}

Contextualization of psychological tests in specific courses is necessary to obtain relevant information to sustain individual or group interventions, unlike general assessment instruments, concentrated in overall academic life. Academic procrastination is relevant, and its evaluation is necessary, due to its short- and medium-term consequences. The objective of the present methodological report was to compare the results of the structural analysis, relationship between dimensions, reliability and incorrect specifications of the Academic Procrastination Scale, evaluated in its general form (sample 1: 717 students, $74.3 \%$ women), and demarcated in specific signatures (sample 2: 142 students, $72.5 \%$ women). Results indicate that the specific form has greater psychometric backing, both in the overall fit, relationship between factors, and lower frequency of erroneous specifications. However, reliability coefficients do not differ between versions. Implications of the results are discussed.

Keywords: academic procrastination; validity; reliability; erroneous specifications; specific signatures.

If the model is truly a good model in terms of its fit in the population, we wish to avoid concluding that the model is a bad one. Alternatively, if the model is truly a bad one, we wish to avoid concluding that it is a good one (MacCallum, Browne, \& Sugawara, 1996; p. 131).

El análisis estructural de un instrumento de evaluación psicológica es un paso fundamental en el proceso de obtención de evidencias de validez. Tradicionalmente es evaluado mediante la técnica del análisis factorial (sea confirmatorio o exploratorio), y sus resultados son capaces de brindar evidencia robusta respecto al grado de influencia del constructo (factores) sobre los indicadores observables (ítems). Ello permitiría al investigador utilizar las puntuaciones del instrumento en diferentes contextos, sea de investigación básica, aplicada, o incluso

${ }^{1}$ Endereço para correspondência: Instituto de Investigación de Psicología, Universidad de San Martín de Porres. Avenida Tomás Marsano, 242, 5 piso, Lima 34, Perú. E-mails: sdominguezmpcs@gmail.com; sdominguezl@usmp.pe 
en su actuación profesional, como una representación objetiva del constructo evaluado. Sin embargo, las fuentes de error son frecuentes y pueden afectar la estructura interna del instrumento, así como la relación entre factores, en el caso de las escalas multidimensionales, manifestándose de diferentes modos (bajas comunalidades, residuales correlacionados, cargas cruzadas, complejidad no modelada, entre otros). Todo ello podría llevar al investigador a concluir de forma errada sobre la estructura factorial de dicho instrumento.

En este punto pueden considerarse dos situaciones asociadas a algunas dificultades en el análisis psicométrico de un instrumento: (a) la de especificidad/amplitud conceptual del constructo evaluado, y (b) la diversidad de contextos/escenarios en base a los que una persona responde un instrumento de evaluación.

En el primer caso, se observa que algunos constructos amplios presentan una baja correlación empírica con constructos más específicos. Por ejemplo, algunos estudios sugieren que no existe relación significativa entre autoeficacia general y autoeficacia académica (Blanco, 2010), ya que si bien parten de una base teórica común, la persona actúa en contextos diferentes y en cada una de ellas podría mostrar un menor o mayor grado de autoeficacia dependiendo de su competencia percibida en dicha área. De igual manera, la relación observada de la procrastinación académica (PA) con la procrastinación general fue cercana a cero en estudiantes peruanos (Álvarez, 2010). Este punto, una alternativa viable sería centrar la atención en un contexto/situación específica y valorar el constructo en base a este.

El segundo caso es más relevante en el ámbito de la evaluación en educación superior por las potenciales consecuencias a nivel de planes tutoriales e intervención educativa. El desempeño de los estudiantes no es uniforme en cada asignatura, así como las estrategias necesarias para lograr el éxito académico, los hábitos académicos empleados, y el grado de exigencia (Dominguez-Lara, Calderón-De la Cruz, Alarcón-Parco, \& Navarro-Loli, 2017; Everson, Tobias, Hartman, \& Gourgey, 1993; Sapp, Farrel, \& Durand, 1995), además del estilo de enseñanza del docente (normalmente, uno por cada asignatura). Por ello, al responder un instrumento de carácter general (p.e., autoeficacia académica, procrastinación académica, etc.), que solicita información global sobre la conducta académica del examinado, podrían existir algunos inconvenientes.

Por ejemplo, al tratar con el constructo PA podrá visualizarse mejor el panorama. La PA se refiere a la acción de retrasar voluntaria e innecesariamente la realización de tareas al punto de experimentar malestar subjetivo (Solomon \& Rothblum, 1984; Steel, 2007). En el contexto universitario peruano es evaluada con la Escala de Procrastinación Académica (EPA; Álvarez, 2010; Dominguez-Lara, 2014, 2016a) que consta de dos dimensiones: Postergación de actividades, y Autorregulación académica; congruentes con los avances teóricos actuales (Grunschel, Patrzek, \& Fries, 2013; Klingsieck, Grund, Schmid, \& Fries, 2013; Steel \& Klingsieck, 2016). Entonces, al administrar la EPA, el evaluado podría basar las respuestas de algunos ítems en un curso, y para contestar otros ítems consideraría su conducta en una asignatura distinta. De ese modo, coexistirían respuestas de postergación y de autorregulación a diferentes asignaturas, lo que sería congruente desde un punto de vista general, ya que quizás responda los ítems de la dimensión postergación pensando en el curso menos agradable, y las de la dimensión autorregulación en función de aquella asignatura que lo motive más, dada la influencia del clima de aula sobre la conducta procrastinadora (Corkin, Shirley, Wolters, \& Wiesner, 2014). Eso fue evidente en el estudio normativo de la EPA en universitarios limeños, donde la correlación entre las dimensiones fue bastante baja (Dominguez-Lara, 2016a), pese a que teóricamente se esperaba una relación empírica de mayor magnitud.

La PA se halla presente en el $14.1 \%$ de estudiantes universitarios peruanos (Dominguez-Lara, en prensa), y su estudio es importante dada su relación inversa con el rendimiento académico (Kim \& Seo, 2015; Kim, Fernandez, \& Terrier, 2017), autoeficacia académica (Dominguez-Lara, en prensa; Wäschle, Allgaier, Lachner, Fink, \& Nückles, 2014) y la motivación académica (Burnam, Komarraju, Hamel, \& Nadler, 2014), lo que podría afectar sobremanera la permanencia del estudiante en el recinto universitario y facilitar de ese modo la deserción estudiantil, dada su estrecha relación con las conductas orientadas al logro (Wu \& Fan, 2017). Sin embargo, no existe evidencia concluyente sobre la influencia de la satisfacción con los estudios sobre la PA, medida con la EPA (Dominguez-Lara, \& CamposUscanga, 2017).

En estos casos, las conclusiones derivadas de la evaluación no brindarían información de utilidad que pueda ser usada en planes de tutoría, ya que por lo general algunos cursos se perciben con mayor dificultad que otros y las tasas de desaprobados son significativamente más elevadas, como las observada en los cursos vinculados a la estadística y metodología de la investigación en psicología (Blanco, 2008; Freng, Webber, Blatter, Wing, \& Scott, 2015; Zeidner, 1991). Por el contrario, si la intención es obtener una vista general de un grupo, independientemente de la asignatura, no acarrearía mayores dificultades, pero es evidente que la valoración por asignatura brindaría información más completa.

Entonces, ante la necesidad de obtener información relacionada con cada asignatura, sería conveniente consultar al evaluado sobre su desempeño en la misma, y de ese modo obtener un reporte más útil para fines institucionales y, en última instancia, para decisiones personales. Para ello, podrían reformularse las instrucciones (focalizarlas en la asignatura) y ligeros cambios al fraseo de los ítems. Sin embargo, la modificación del instrumento 
debe ser evaluada psicométricamente, ya que si no mejoran significativamente las propiedades psicométricas (confiabilidad y validez), sobre todo la dimensionalidad, entonces dicha modificación sería irrelevante, y podría seguir empleándose la forma general. Por ello, es necesario evaluar si existe algún efecto sobre las propiedades psicométricas de un instrumento cuando se le solicita al estudiante que responda por una asignatura en concreto (forma específica), con relación a la forma general, donde se le consulta habitualmente sobre su vida académica.

De este modo, el objetivo del presente reporte metodológico fue comparar los indicadores asociados a la estructura interna, relación entre dimensiones, y confiabilidad de la EPA-general y EPA-específica. Además, serán evaluadas las malas especificaciones (ME; Hu \& Bentler, 1998; MacCallum et al., 1996) al interior de cada modelo, cuya aplicación no tiene precedentes en el ámbito latinoamericano, y es un aspecto necesario en el marco de toma de decisiones respecto al modelo que mejor representan los datos.

Existe una ME cuando uno o más parámetros son especificados aun cuando sus valores poblacionales son cero (sobre-parametrización), o cuando algunos parámetros son fijados a cero, pero sus valores poblacionales son diferentes de cero (infra-parametrización) ( $\mathrm{Hu}$ \& Bentler, 1998). Esto tiene completa relevancia para el presente trabajo ya que se asumiría que ante una equivalencia estructural y de confiabilidad, el modelo que presenta más ME relevantes, sería el peor modelo.

En este punto, es de utilidad el método propuesto por Saris, Satorra y Van der Veld (2009) que busca determinar la relevancia de las ME encontradas a través de los índices de modificación (IM; Sörbom, 1989), analizando el poder estadístico de cado uno de ellos, y el cambio esperado en el parámetro indicado, sobre todo en modelos oblicuos, donde se halla presente gran parte de la complejidad no modelada y los errores correlacionados (Murray, \& Johnson, 2013). De esta forma, se superaría una limitación de las aproximaciones tradicionales que solo valoran los modelos en función de sus índices de ajuste (Dominguez-Lara, 2016b), reteniendo o rechazando modelos, pero sin tener en cuenta indicadores adicionales que ayuden al investigador a tomar una decisión más informada.

\section{Método}

\section{Participantes}

La muestra general (M1) provino de un estudio previo de normalización de la EPA (Dominguez-Lara, 2016a), y estuvo compuesta por 717 estudiantes universitarios de psicología: $74.3 \%$ mujeres, $M_{\text {edad }}=22.65,33.5 \%$ trabaja, $92.2 \%$ es soltero. La muestra específica (M2) se obtuvo de forma intencional, y estuvo formada por 142 estudiantes (72.5\% mujeres), con edades comprendidas entre 18 y 42 años $(M=22.60 ; D E=3.472)$, quienes cursaban el quinto (dos aulas), séptimo (un aula) y octavo ciclo (dos aulas) de la carrera de psicología en una universidad privada de Lima Metropolitana.

\section{Instrumento}

Escala de Procrastinación Académica (EPA; Busko, 1998). Fue utilizada una versión variante de la adaptación a universitarios limeños (Dominguez-Lara, Villegas, \& Centeno, 2014) focalizada en un curso específico. Consta de dos dimensiones, Postergación de actividades (tres ítems) y Autorregulación académica (nueve ítems). Los ítems cuentan con cinco opciones de respuesta (Nunca, Pocas veces, A veces, Casi siempre, Siempre). La interpretación de los puntajes es directa: a mayor puntaje, mayor presencia de la conducta evaluada.

\section{Procedimiento}

\section{Cambios en el Fraseo}

La instrucción original de la EPA es: "A continuación encontrarás una serie de preguntas que hacen referencia a tu modo de estudiar. Lee cada frase y contesta según tus últimos 12 meses de tu vida como estudiante marcando con una X de acuerdo con la siguiente escala de valoración"; y fue cambiada colocando en el encabezado del test la frase "Con respecto a este curso..." seguido por "A continuación encontrarás una serie de preguntas que hacen referencia a tu modo de estudiar. Lee cada frase y contesta marcando con una $\mathrm{X}$ de acuerdo con la siguiente escala de valoración". A su vez en las preguntas seis y siete fue omitida la frase "de los cursos que no me gustan" y fue cambiada por "del curso" (p.e., el contenido del ítem 6 fue "Postergo los trabajos del curso"). Debido a que los cambios fueron mínimos, para evaluar la claridad solo fueron consultados cinco estudiantes de pregrado de otra universidad privada. No se evidenciaron inconvenientes en cuanto a la comprensión de los enunciados.

\section{Aplicación de Instrumentos}

El proyecto fue aprobado por la Dirección del Instituto de Investigación de Psicología de la universidad participante, donde fueron verificados los aspectos éticos y procedimentales de la investigación.

La aplicación de la versión modificada del EPA a M2 fue llevada a cabo por los docentes encargados de las asignaturas (cuatro en total). Dicha evaluación fue realizada inmediatamente después del examen parcial, previo consentimiento verbal del estudiante. La EPA se hallaba al interior de una batería de instrumentos en el marco del desarrollo del proyecto mencionado en la página inicial de este manuscrito.

Preliminarmente fueron explicados los objetivos de la investigación resaltando el carácter voluntario de la participación, y que ésta no sería recompensada académica o económicamente. Durante la aplicación de los instrumentos a M2, se les solicitó que respondan algunos de 
ellos (p.e., la EPA) pensando en la asignatura en la que recientemente habían sido evaluados. Cabe precisar la versión aplicada a M1 indicaba que respondan pensando en los últimos doce meses de vida estudiantil (DominguezLara, 2016a).

\section{Tratamiento Estadístico de los Datos}

De forma preliminar, fue calculado el coeficiente de Mardia (1970) como indicador de normalidad multivariada, esperando magnitudes menores que 70 (Rodriguez \& Ruiz, 2008) para acreditar tal condición. En cuanto a M1, fue utilizada la información sobre el análisis estructural y de confiabilidad de la EPA procedente del estudio de normalización en universitarios limeños (DominguezLara, 2016a). Solo fueron re-calculados los coeficientes de confiabilidad y los IM.

Respecto a M2, la estructura de dos factores oblicuos de la EPA fue analizada utilizando el programa EQS 6.2 (Bentler \& Wu, 2012) bajo las siguientes condiciones: método de máxima verosimilitud, matriz de correlaciones policóricas (Dominguez-Lara, 2014; Lee, Poon, \& Bentler, 1995) y estadísticos robustos. El ajuste del modelo fue evaluado utilizando la prueba SB- $\chi^{2}$ (Satorra \& Bentler, 1994), RMSEA ( $\leq .05)$, CFI ( $\geq .95)$, y el SRMR $(\leq .08)$ de forma conjunta, así como el criterio de información de Akaike (AIC; 1987), cuyo valor más baja hace referencia al modelo más parsimonioso. La validez interna convergente fue evaluada por medio de la fuerza de asociación entre las dos dimensiones que componen la EPA: Postergación de Actividades, y Autorregulación Académica; esperando una relación inversa atendiendo al significado teórico de las escalas. De forma complementaria, fueron estimados los IM (Sörbom, 1989) asociados a residuales correlacionados y cargas cruzadas, y con ellos determinar si existen ME relevantes con los datos de M1 y M2. Por otro lado, para el análisis de la confiabilidad, fueron implementados los coeficientes $\omega$ (McDonald, 1999), H (Dominguez-Lara, 2016c; Hancock \& Mueller, 2001), y $\alpha$ (Cronbach, 1951).

Los indicadores resultantes de los análisis realizados en M1 y M2 fueron comparados de diferente manera. Con relación al ajuste del modelo, fueron considerados aquellos índices de ajuste relacionados con la complejidad (p.e., RMSEA, AIC). En cuanto a la confiabilidad, los coeficientes $\alpha$ de cada dimensión (Postergación de Actividades, y Autorregulación Académica) obtenidos en M1 y M2 fueron comparados estadísticamente mediante el método de Feldt (Feldt, 1980; Feldt, Woodruff, \& Salih, 1987).

Para comparar las correlaciones interfactoriales entre las dimensiones de la EPA halladas en M1 y M2 fue usado el método general asimétrico basado en el intervalo de confianza (IC) para la diferencia entre correlaciones (Zou, 2007). La valoración es sencilla: si el IC de la diferencia contiene al cero, la diferencia no es estadísticamente significativa.
Finalmente, con relación a la evaluación de las ME, se dice que estas son relevantes cuando: (a) el IM es estadísticamente significativo y está asociado a un bajo poder estadístico, y (b) el IM es estadísticamente significativo, está asociado a un alto poder estadístico, y el Cambio Esperado en el Parámetro (CEP) es mayor que .10. El poder estadístico haría referencia a la sensibilidad para detectar cambios potencialmente significativos en los parámetros. En cambio, si el IM no es estadísticamente significativo y está asociado a un alto poder estadístico, la ME no es relevante. Finalmente, cuando el IM no es estadísticamente significativo y está asociado a un bajo poder estadístico, no existe información suficiente para concluir a favor o en contra de la relevancia de la ME (Para más detalles técnicos del método, ver Saris et al., 2009). El estadístico $\delta$, que expresa el tamaño mínimo de la ME que podría detectarse por la prueba con una alta probabilidad (potencia), fue fijado a .10, valor recomendado para parámetros estructurales estandarizados y errores correlacionados (Saris et al., 2009). Para este procedimiento fue empleada una sintaxis en SPSS ad hoc, disponible al lector sin costo alguno. Por último, ante la ausencia de índices estadísticos para comparar cantidad de ME presentes, solo serán contabilizadas en cada muestra (M1 y M2), analizando además las características de las mismas (residuales correlacionados o cargas cruzadas).

\section{Resultados}

La normalidad multivariada evaluada con el coeficiente de Mardia brinda indicadores adecuados tanto para M1 (39.701) como para M2 (28.928). El análisis estructural de la EPA en M2 indica un mejor ajuste, con relación a $\mathrm{M} 1$, sobre todo en lo que concierne al ajuste general $\left(\mathrm{SB}-\chi^{2}\right)$. Asimismo, existe menos discrepancia entre el modelo observado y el modelo teórico en M2 que en M1 $\left(\mathrm{SRMR}_{1}>\mathrm{SRMR}_{2}\right)$ (Tabla 1$)$.

En cuanto a la validez interna convergente, la relación conceptual entre Postergación y Autorregulación es más fuerte y va en la dirección esperada (inversa) cuando la evaluación se focaliza en la asignatura (M2), comparada con la evaluación general (M1), donde la relación entre dimensiones es bastante baja (Tabla 1). Además, el IC para la diferencia entre $\phi_{1} y \phi_{2}$ fue de $(-.640,-.397)$.

Por el contrario, no hubo mejora significativa en cuanto a la confiabilidad de las puntuaciones evaluada con el coeficiente $\alpha$ para la dimensión Postergación $\left(\chi_{[1]}^{2}=3.782, p>.05\right)$, e inclusive parece que el formato de evaluación general favorece la consistencia de la dimensión Autorregulación Académica $\left(\chi_{[1]}^{2}=7.790\right.$, $p<.05)$. Un panorama similar se observa con el coeficiente $\omega$, donde en apariencia no hubo diferencias en cuanto a su magnitud (Tabla 1). Ello indicaría que el error de medición se mantiene constante entre las muestras, lo que podría esperarse ya que todos son estudiantes de psicología. 
Tabla 1

Análisis Estructural y de Confiabilidad de la EPA en M1 y M2

\begin{tabular}{|c|c|c|}
\hline & M1 $(n=717)$ & M2 $(n=142)$ \\
\hline \multicolumn{3}{|l|}{ Análisis estructural } \\
\hline $\mathrm{SB}-\chi^{2}{ }_{(53)}[p>.05]$ & $204.976(p<.001)$ & $56.053(p=.361)$ \\
\hline CFI $[\geq .95]$ & .974 & .996 \\
\hline RMSEA (IC 90\%) [ $\leq .05]$ & $.063(.054-.072)$ & $.020(.000-.058)$ \\
\hline SRMR $[\leq .05]$ & .071 & .061 \\
\hline AIC & 98.976 & -.49 .947 \\
\hline$\phi$ & -.118 & -.644 \\
\hline \multicolumn{3}{|l|}{ Análisis de confiabilidad } \\
\hline$\alpha$ Postergación & .754 & .831 \\
\hline$\alpha$ Autorregulación & .868 & .805 \\
\hline$\omega$ Postergación & .811 & .835 \\
\hline$\omega$ Autorregulación & .892 & .796 \\
\hline
\end{tabular}

Nota. Entre corchetes: puntos de corte idóneos; $\phi$ : correlación interfactorial

Una vez solicitados los IM asociados a parámetros que podrían ser especificados para mejorar el ajuste del modelo (correlación entre residuales, y cargas cruzadas), con M1 fueron encontrados 24 pares de errores correlacionados estadísticamente significativos (ninguna carga cruzada), y aunque solo seis de ellos presentan $\mathrm{CEP}>.10$, fueron evaluados todos; mientras que con M2, solo fueron cinco pares de errores correlacionados estadísticamente significativos. No fueron analizados los IM sin significancia estadística ya que es poco probable encontrar ME asociados a ellos (Saris et al., 2009).

Un análisis individual indica que para M1 seis de los 24 IM se asocian a una ME (Tabla 2); por el contrario, en M2 solo un IM fue asociado a una ME, y no hay información suficiente para uno de ellos (Tabla 3).

Tabla 2

Análisis de las ME en M1

\begin{tabular}{|c|c|c|c|c|c|}
\hline Errores correlacionados & IM & CEP (IC95\%) & PNC & Poder & Decisión \\
\hline Ítem 2 y 4 & 57.197 & $-.199(-.251,-.147)$ & 14.443 & .967 & CEP: M \\
\hline Ítem 2 y 11 & 34.157 & $.157(.104, .157)$ & 13.857 & .961 & CEP: M \\
\hline Ítem 4 y 11 & 33.111 & $.117(.077, .117)$ & 24.188 & .998 & CEP: M \\
\hline Ítem 1 y 2 & 28.947 & $-.176(-.240,-.112)$ & 9.345 & .864 & CEP: M \\
\hline Ítem 2 y 9 & 27.941 & $.153(.096, .210)$ & 11.936 & .933 & CEP: M \\
\hline Ítem 4 y 5 & 20.593 & $.084(.048, .120)$ & 29.185 & 1.000 & CEP: NM \\
\hline Ítem 6 y 7 & 20.179 & $2.031(1.145,2.031)$ & .049 & .056 & $\mathrm{M}$ \\
\hline Ítem 6 y 8 & 16.627 & $.059(.031, .087)$ & 47.765 & 1.000 & CEP: NM \\
\hline Ítem 5 y 10 & 15.463 & $-.066(-.099,-.033)$ & 35.498 & 1.000 & CEP: NM \\
\hline Ítem 9 y 12 & 13.891 & $-.081(-.124,-.038)$ & 21.172 & .996 & CEP: NM \\
\hline Ítem 4 y 7 & 13.780 & $.059(.028, .090)$ & 39.586 & 1.000 & CEP: NM \\
\hline Ítem 1 y 11 & 11.596 & $-.084(-.132,-.036)$ & 16.434 & .982 & CEP: NM \\
\hline Ítem 11 y 12 & 11.292 & $.068(.028, .108)$ & 24.420 & .999 & CEP: NM \\
\hline Ítem 9 y 10 & 11.116 & $.064(.026, .102)$ & 27.139 & .999 & CEP: NM \\
\hline Ítem 1 y 10 & 11.050 & $.069(.028, .110)$ & 23.209 & .998 & CEP: NM \\
\hline Ítem 9 y 11 & 10.587 & $.072(.029, .115)$ & 20.422 & .995 & CEP: NM \\
\hline Ítem 3 y 12 & 10.324 & $.068(.027, .109)$ & 22.327 & .997 & CEP: NM \\
\hline Ítem 3 y 8 & 9.868 & $-.062(-.101,-.023)$ & 25.671 & .999 & CEP: NM \\
\hline Ítem 4 y 9 & 8.363 & $-.063(-.106,-.020)$ & 21.071 & .996 & CEP: NM \\
\hline Ítem 7 y 8 & 7.891 & $-.041(-.070,-.012)$ & 46.942 & 1.000 & CEP: NM \\
\hline Ítem 8 y 11 & 6.801 & $-.049(-.086,-.012)$ & 28.326 & 1.000 & CEP: NM \\
\hline
\end{tabular}


Tabla 2 (continuación) Análisis de las ME en M1

\begin{tabular}{cccccc}
\hline Errores correlacionados & IM & CEP (IC95\%) & PNC & Poder & Decisión \\
\hline Ítem 7 y 10 & 6.729 & $.036(.009, .063)$ & 51.921 & 1.000 & CEP: NM \\
Ítem 2 y 10 & 4.648 & $-.049(-.094,-.004)$ & 19.359 & .993 & CEP: NM \\
Ítem 5 y 7 & 4.361 & $-.030(-.058,-.002)$ & 48.456 & 1.000 & CEP: NM \\
\hline
\end{tabular}

Nota. IM=índice de modificación; $\mathrm{CEP}=$ cambio esperado en el parámetro; $\mathrm{PNC}=$ parámetro de no centralidad; $\mathrm{M}=$ mala especificación detectada; $\mathrm{NM}=$ mala especificación no detectada; $\mathrm{CEP}: \mathrm{M}=\mathrm{M}$ detectada a partir de la inspección del CEP; CEP: NM=NM detectada a partir de la inspección del CEP

Tabla 3

Análisis de las ME en M2

\begin{tabular}{ccccccc}
\hline Errores correlacionados & IM & CEP (IC95\%) & PNC & Poder & Decisión \\
\hline Ítem 3 y 5 & 8.934 & $-.182(-.301,-.063)$ & 2.697 & .376 & CEP: NM \\
Ítem 3 y 12 & 7.903 & $.197(.060, .334)$ & 2.036 & .297 & M \\
Ítem 1 y 2 & 6.897 & $-.158(-.276,-.040)$ & 2.763 & .383 & CEP: NM \\
Ítem 5 y 11 & 5.950 & $.138(.027, .249)$ & 3.124 & .424 & CEP: NM \\
Ítem 4 y 12 & 4.369 & $.148(.009, .287)$ & 1.995 & .292 & I \\
\hline
\end{tabular}

Nota. IM=índice de modificación; $\mathrm{CEP}=$ cambio esperado en el parámetro; $\mathrm{PNC}=$ parámetro de no centralidad; $\mathrm{M}=$ mala especificación detectada; CEP: NM=M no detectada a partir de la inspección del CEP; I=No existe información suficiente para determinar la presencia de una mala especificación

\section{Discusión}

El objetivo fue comparar los indicadores asociados a la estructura interna, relación entre dimensiones, confiabilidad, y malas especificaciones de la EPA-general (M1) y EPA-específica (M2). Los resultados mostrados indicarían que la dimensionalidad de la EPA parece más robusta en $\mathrm{M} 2$, es decir, la forma más específica. A ello se le agrega las magnitudes exhibidas de los estadísticos relacionados con la complejidad del modelo, como el RMSEA, cuya puntuación a favor de M2 indica que mucha de la complejidad no modelada desaparece cuando las respuestas van en función de una sola asignatura, siendo además más parsimonioso a juzgar por su índices específicos (AIC1 > AIC2). Si bien podría argumentarse que el tamaño muestral podría favorecer a M2, las magnitudes del CFI (aceptable en M1 y M2) indican que ese aspecto ser relevante en la decisión final. Probablemente, a ello se deba que tanto el CFI como el RMSEA tengan mejor performance en M2, ya que esos índices de ajuste son sensibles a las ME (Chen, 2007), y son poco afectados por el tamaño muestral (Dimitrov, 2010).

Sobre la validez interna convergente de la EPA en sus dos formas (general y específica), quizás el indicador más fuerte de diferenciación sea la comparación de las correlaciones entre las dimensiones, donde fueron halladas diferencias significativas entre ambas versiones. Sin embargo, la estimación del error de medición se mantiene constante entre M1 y M2.

Concluyendo, y al menos con el instrumento evaluado, contextualizar a una asignatura en lugar de realizar una valoración general de la conducta del estudiante brinda propiedades psicométricas más aceptables en términos de: (a) un modelo más parsimonioso, (b) relaciones empíricas entre dimensiones teóricamente congruentes, y (c) menor cantidad de ME asociadas a los IM.

Respecto a las ME, la existencia de IM significativos asociados a un número mayor de residuales correlacionados en M1 sugiere la presencia de una fuente más grande de varianza irrelevante a los constructos estudiados, en contraste con M2. Como mencionó inicialmente, ello podría estar determinado por la falta de especificidad de la EPA en su forma general al evocar respuestas de diferente fuente (e.g., asignaturas) y, por ende, el modelo verdadero no sería el mismo que el de la EPA en su forma específica.

Con todo, la EPA sería una buena opción evaluativa en contextos específicos de cada asignatura, tanto individual, para conocer cómo es el desempeño de un estudiante con relación a los demás, así como grupal, ya que podrían ser comparados los promedios de diversas asignaturas evaluados al interior de un salón de clases. Sin embargo, es necesario mencionar que el modelo que subyace al EPA en su forma específica es más congruente desde un punto de vista teórico que la general.

Entre las limitaciones del estudio destaca el desbalance entre el tamaño muestral de los grupos, pero pese a ello los indicadores fueron robustos. Además, ante el cumplimiento del supuesto de normalidad multivariada y la homogeneidad de la muestra (todos estudiantes de psicología), no parece una limitación tan grave, pero la ampliación a otras carreras profesionales podría ser una 
opción válida debido a la variedad de asignaturas que afronta el estudiante en cada una de ellas.

Definitivamente, es necesario que en futuras investigaciones puedan valorarse de forma conjuntan ambas formas, es decir, evaluarlas en el mismo grupo, y de ese modo analizar su capacidad predictiva individual con relación a aspectos relevantes vinculados al ámbito académico, principalmente el rendimiento. Asimismo, es relevante implementar una comparación de correlaciones con relación a criterios externos para brindar evidencias adicionales sobre la validez de constructo. Por ejemplo, se podrían relacionar las puntuaciones de una escala de autoeficacia académica (AA) focalizada en la asignatura, con la EPA-general $\left(r_{1}\right)$, y esas puntuaciones de AA con la EPA-específica $\left(r_{2}\right)$, y luego de ello comparar las correlaciones con métodos vistos en este trabajo $\left(\mathrm{IC}_{r 1}-{ }_{r 2}\right.$; Zou, 2007), hipotetizando la existencia de diferencias significativas entre correlaciones $\left(r_{2}>r_{1}\right)$. Del mismo modo, analizar la influencia de otras variables relacionadas al ámbito académico según el sexo, ya que es probable que aparezcan algunas diferencias importantes (DominguezLara \& Campos-Uscanga, 2017).

\section{Consideraciones Éticas}

Protección de personas: Los autores declaran que para esta investigación no se han realizado experimentos en seres humanos ni en animales.

Confidencialidad de los datos: Los autores declaran que han seguido los protocolos de su centro de trabajo sobre la publicación de datos personales.

Derecho a la privacidad y consentimiento informado: el autor han obtenido el asentimiento informado de los sujetos referidos en el artículo, quienes respondieron con el conocimiento de que colocarían sus datos de identificación.

Conflicto de intereses: El autor declara no tener conflictos de intereses.

\section{Referencias}

Akaike, H. (1987). Factor analysis and AIC. Psychometrika, 52(3), 317-332. doi: 10.1007/978-1-4612-1694-0 29

Álvarez, O. (2010). Procrastinación general y académica en una muestra estudiantes de secundaria de Lima. Persona, 13, 159-177. Recuperado de http://www.redalyc.org/pdf/1471/147118212009.pdf

Bentler, P. M., \& Wu, E. J. C. (2012). EQS 6.2 for Windows [Statistical Program]. Encino, CA: Multivariate Software, Inc.

Blanco, Á. (2008). Una revisión crítica de la investigación sobre las actitudes de los estudiantes universitarios hacia la estadística. Revista Complutense de Educación, 19(2), 311-330.

Blanco, A. (2010). Creencias de autoeficacia de estudiantes universitarios: Un estudio empírico sobre la especificidad del constructo. Revista Electrónica de Investigación y Evaluación Educativa, 16(1), 1-28.

Burnam, A., Komarraju, M., Hamel, R., \& Nadler, D. R. (2014). Do adaptive perfectionism and self-determined motivation reduce academic procrastination? Learning and Individual Differences, 36, 165-172. doi: 10.1016/j.lindif.2014.10.009

Busko, D. (1998). Causes and consequences of perfectionism and procrastination: A structural equation model. (Tesis de Maestría no publicada). The University of Guelph: Canadá.

Chen, F. F. (2007). Sensitivity of goodness of fit indexes to lack of measurement invariance. Structural Equation Modeling, 14(3), 464-504. doi: $10.1080 / 10705510701301834$

Corkin, D. M., Shirley, L. Y., Wolters, C. A., \& Wiesner, M. (2014). The role of the college classroom climate on academic procrastination. Learning and Individual Differences, 32, 294-303. doi: 10.1016/j.lindif.2014.04.001

Cronbach L. J. (1951). Coefficient alpha and the internal structure of tests. Psychometrika, 16(3), 297-334. doi: 10.1007/BF02310555

Dimitrov, D. M. (2010). Testing for factorial invariance in the context of construct validation. Measurement and Evaluation in Counseling and Development, 43(2), 121-149. doi: 10.1177/0748175610373459

Dominguez-Lara, S. (en prensa). Prevalencia de procrastinación académica en estudiantes universitarios de Lima Metropolitana y su relación con variables demográficas. Revista de Psicología-UCSP.

Dominguez-Lara, S. (2014). ¿Matrices Policóricas/Tetracóricas o Matrices Pearson? Un estudio metodológico. Revista Argentina de Ciencias del Comportamiento, 6(1), 39-48.

Dominguez-Lara, S. (2016a). Datos normativos de la Escala de Procrastinación Académica en estudiantes de psicología de Lima. Evaluar, 16(1), 20-30.

Dominguez-Lara, S. (2016b). Evaluación de modelos estructurales, más allá de los índices de ajuste. Enfermería Intensiva, 27(2), 84-85.

Dominguez-Lara, S. (2016c). Evaluación de la confiabilidad del constructo mediante el Coeficiente H: Breve revisión conceptual y aplicaciones. Psychologia: Avances en la Disciplina, 10(2), 84-94.

Dominguez-Lara, S., \& Campos-Uscanga, Y. (2017). Influencia de la satisfacción con los estudios sobre la procrastinación académica en estudiantes de psicología: Un estudio preliminar. Liberabit, 23(1),123-135. doi: 10.24265/liberabit.2017.v23n1.09

Dominguez-Lara, S., Villegas, G., \& Centeno, S. (2014). Procrastinación académica: Validación de una escala en una muestra de estudiantes de una universidad privada. Liberabit, 20(2), 293-304.

Dominguez-Lara, S., Calderón-De la Cruz, G., Alarcón-Parco, D., \& Navarro-Loli, J. (2017). Relación entre Ansiedad ante exámenes y rendimiento en exámenes en universitarios: Análisis preliminar de la diferencia según asignatura. Revista Digital de Investigación en Docencia Universitaria, 11(1), 123-135. doi: 10.19083/ridu.11.492

Everson, H. T., Tobias, S., Hartman, H., \& Gourgey, A. (1993). Test anxiety and the curriculum: The subject matters. Anxiety, Stress and Coping: An International Journal, 6(1), 1-8. doi: 10.1080/10615809308249528 
Feldt, L. S. (1980). A test of the hypothesis that Cronbach's alpha reliability coefficient is the same for two tests administered to the same sample. Psychometrika, 48(1), 99-105. doi: 10.1007/BF02293600

Feldt, L. S., Woodruff, D. J., \& Salih, F. A. (1987). Statistical inference for coefficient alpha. Applied Psychological Measurement, 11(1), 93-103. doi: $10.1177 / 014662168701100107$

Freng, S., Webber, D., Blatter, J., Wing, A., \& Scott, W. D. (2015). The role of statistics and research methods in the academic success of psychology majors: Do performance and enrollment timing matter? Teaching of Psychology, 32(2), 83-88. doi: 10.1177/0098628311401591

Grunschel, C., Patrzek, J., \& Fries, S. (2013). Exploring reasons and consequences of academic procrastination: An interview study. European Journal of Psychology of Education, 28(3), 841-861. doi: 10.1007/s10212-012-0143-4

Hancock, G. R., \& Mueller, R. O. (2001). Rethinking construct reliability within latent variable systems. In R. Cudeck, S. H. C. du Toit, \& D. Sörbom (Eds.), Structural equation modeling: Past and present. A Festschrift in honor of Karl G. Jöreskog (pp. 195-261). Chicago: Scientific Software International.

Hu, L., \& Bentler, P. M. (1998). Fit índices in covariance structure modeling: Sensitivity to underparameterized model specification. Psychological Methods, 3(4), 424-453.

Kim, K. R., \& Seo, E. H. (2015). The relationship between procrastination and academic performance: A meta-analysis. Personality and Individual Differences, 82, 26-33. doi: 10.1016/j.paid.2015.02.038

Kim, S., Fernandez, S., \& Terrier, L. (2017). Procrastination, personality traits, and academic performance: When active and passive procrastination tell a different story. Personality and Individual Differences, 108, 154-157. doi: 10.1016/j.paid.2016.12.021

Klingsieck, K. B., Grund, A., Schmid, S., \& Fries, S. (2013). Why students procrastinate: A qualitative approach. Journal of College Student Development, 54(4), 397-412. doi: 10.1353/csd.2013.0060

Lee, S. Y., Poon, W. Y., \& Bentler, P. M. (1995). A two-stage estimation of structural equation models with continuous and polytomous variables. British Journal of Mathematical and Statistical Psychology, 48(2), 339-358. doi: 10.1111/j.2044-8317.1995.tb01067.x

MacCallum, R. C., Browne, M. W., \& Sugawara, H. M. (1996). Power analysis and determination of sample size for covariance structure modeling. Psychological Methods, 1(2), 130-149.

Mardia, K. (1970). Measures of multivariate skewness and kurtosis with applications. Biometrika, 57(3), 519-530. doi: 10.1093/biomet/57.3.519

McDonald R. P. (1999). Test theory: A unified treatment. Mahwah, N. J. L. Erlbaum Associates.

Murray A. L., \& Johnson, W. (2013). The limitations of model fit in comparing the bi-factor versus high-order models of human cognitive ability structure. Intelligence, 41(5), 407-422. doi: 10.1016/j.intell.2013.06.004

Rodriguez, M., \& Ruiz, M. (2008). Atenuación de la asimetría y de la curtosis de las puntuaciones observadas mediante transformaciones de variables: Incidencia sobre la estructura factorial. Psicológica, 29(2), 205-227.

Sapp, M., Farrel, W., \& Durand, H. (1995). The effects of mathematics, reading, and writting tests in producing worry and emotionality test anxiety with economically and educational disadvantages college students. College Student Journal, 29(1), 122-125.

Saris, W. E., Satorra, A., \& van der Veld, W. M. (2009). Testing structural equation modeling or detection of misspecifications? Structural Equation Modeling, 16(4), 561-582. doi: 10.1080/10705510903203433

Satorra, A., \& Bentler, P. M. (1994). Corrections to test statistics and standard errors in covariance structure analysis. In A. von Eye \& C. C. Clogg (Eds.), Latent variables analysis: Applications for developmental research (pp. 399-419). Thousand Oaks, CA: Sage.

Steel, P., \& Klingsieck, K. B. (2016). Academic Procrastination: Psychological Antecedents Revisited. Australian Psychologist, 51(1), 36-46. doi: 10.1111/ap.12173

Sörbom, D. (1989). Model modification. Psychometrika, 54(3), 371-384. doi: 10.1007/BF02294623

Solomon, L. J., \& Rothblum, E. D. (1984). Academic procrastination: Frequency and cognitive-behavioral correlates. Journal of Counseling Psychology, 31(4), 503-509.

Steel, P. (2007). The nature of procrastination: A meta-analytic and theoretical review of quintessential self-regulatory failure. Psychological Bulletin, 133(1), 65-94.

Wäschle, K., Allgaier, A., Lachner, A., Fink, S., \& Nückles, M. (2014). Procrastination and self-efficacy: Tracing vicious and virtuous circles in self-regulated learning. Learning and Instruction, 29, 103-114. doi: 10.1016/j.learninstruc.2013.09.005

Wu, F., \& Fan, W. (2017). Academic procrastination in linking motivation and achievement-related behaviours: A perspective of expectancyvalue theory. Educational Psychology, 37(6), 695-711. doi: 10.1080/01443410.2016.1202901

Zeidner, M. (1991). Statistics and mathematics anxiety in social science students: Some interesting parallels. British Journal of Educational Psychology, 61(3), 319-328. doi: 10.1111/j.2044-8279.1991.tb00989.x

Zou, G. (2007). Toward using confidence intervals to compare correlations. Psychological Methods, 12(4), 39-413.

\section{Sobre o autor}

Sergio Dominguez-Lara es Psicólogo, y magíster en Psicología Clínica y de la Salud y docente del Instituto de Investigación de Psicología (USMP, Perú). 